\title{
ECONOMICAL AND ACCURATE DIGITALLY PROGRAMMABLE DUAL-POLARITY GAIN AMPLIFIER**
}

\author{
PROFESSOR T.S. RATHORE* Visiting Researcher \\ and DR. L.C. JAIN \\ Knowledge-Based Engineering Systems Group School of Electronic Engineering \\ University of South Australia Adelaide, Australia
}

(Received November 28, 1994; in final form December 29, 1994)

A digitally programmable, dual-polarity gain amplifier is proposed. It uses total resistance less than those presented earlier. The effect of on-resistance of switches can be fully compensated.

\section{INTRODUCTION}

Some amplifiers that can be programmed for dual-polarity with a single switch, and use minimum number of resistors were presented in [1]. However, in IC fabrication it is preferable to have less total resistance rather than the number of resistors. In this paper, a new amplifier that has not only the facility of programming the polarity of the gain with a single switch, but also possesses total resistance considerably less, is proposed.

\section{PROGRAMMABLE AMPLIFIER CONFIGURATION}

The proposed amplifier configuration is shown in Fig. 1. Gain of the amplifier is:

$$
\frac{V_{0}}{V_{i}}= \begin{cases}K_{-}=-p & \text { when } S \text { closed } \\ K_{+}=\frac{1+p+b / c}{1+d}-p & \text { when } S \text { open }\end{cases}
$$

where

$$
\begin{aligned}
& p=a+b+\frac{a b}{c}, \\
& a=\frac{R_{2}}{R_{1}}, b=\frac{R_{3}}{R_{1}}, c=\frac{R_{4}}{R_{1}}, d=\frac{R_{5}}{R_{6}} .
\end{aligned}
$$

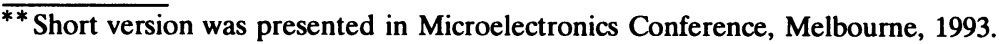

*On leave from IIT, Bombay, India. 


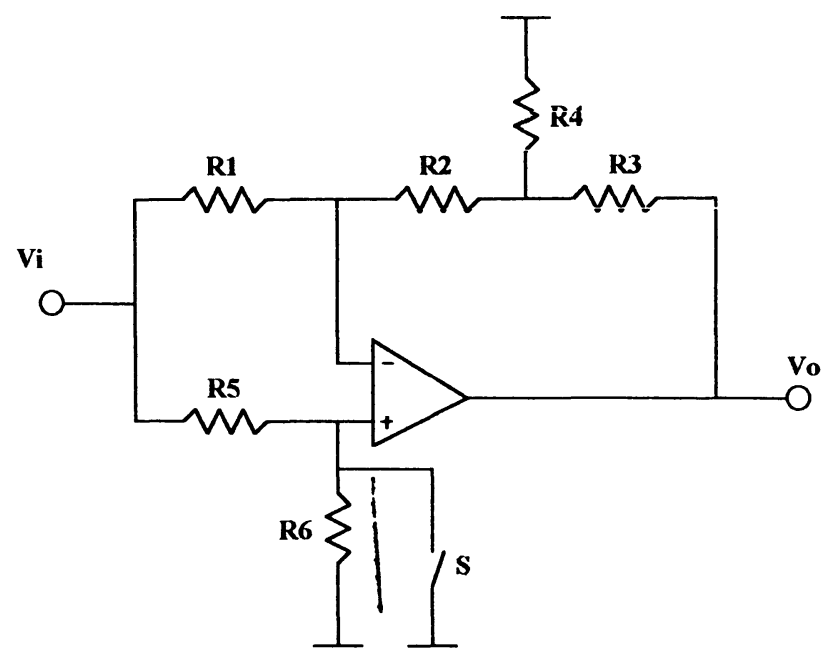

FIGURE 1 Proposed amplifier configuration

It is obvious from eqns (1) and (2) that polarity of the gain can be controlled by the switch $S$. Conditions for $K_{-}=-K_{+}=K$ imposed by eqns. (1) and (2) are

$K=a+b+\frac{a b}{c}$

and

$d=\left(1+\frac{b}{c}-K\right) /(2 K)$

For $d$ to be non-negative, eqn. (5) demands

$\left(1+\frac{b}{c}\right) \geq K$

Since, for finite gain, $1 \leq K<\infty$, eqns (4) and (5) imply

$c \neq 0, a \neq \infty, b \neq \infty ; b \neq 0, c \neq \infty, d \neq \infty$.

Out of many possibilities that satisfy eqns. (4), (5), we consider the following, which reduces one resistor

Case A: $\quad a=0 \quad R_{2}=0$, or $R_{1}=\infty$

Case B: $d=0 \quad R_{5}=0$, or $R_{6}=\infty$.

$R_{1}=\propto$ is not admissible, as it will reduce, see eqn. (3), $b=c=0$, the undesirable conditions in eqn. (7). Also, $R_{5}$ cannot be zero, because when $S$ is closed (for 
negative gains), input will be shorted. However, $R_{5}$ can be replaced by a switch $\bar{S}$, which opens when $S$ is closed and vice versa.

Case A: $R_{2}=0$

In this case the circuit of Fig. 1 reduces to a circuit that has been studied in detail in Ref. [1]. Out of the four cases, case (iv) in [1] restricts the gain $\leq 1$. Being a case of an attenuator (and not an amplifier), it will not be considered.

For the remaining cases, the amplifier circuits are shown in Fig. 2 (a), (b) and (c), where we have modified the resistances so as to yield an input resistance $\geq R$. In Fig. (c), the resistance $R_{4}$ is replaced by a switch $\bar{S}_{1}$, which opens when $S_{1}$ closes and vice versa. The total resistances for a specific gain $K$ for the three circuits are, respectively,

$R_{t a}=6(1+K) R \quad K \geq 0$

$R_{t b}=2\left(4+\frac{2 K^{2}}{2 K-1}\right) R \quad K \geq \frac{1}{2}$

$R_{t c}=\left(1+\frac{K^{2}}{K-1}\right) R \quad K \geq 1$

It can be verified that

$R_{t b}, R_{t c}<R_{t a}, \quad$ for all $K$

$R_{t c} \leq R_{t b}, \quad K=1, K \geq 1.112$

Case B: $R_{6}=\infty, R_{5}$ replaced by a switch $\bar{S}$.

For this case, the design relations are

$$
\begin{aligned}
& R_{1}=R\left(\text { For } R_{\text {in }} \geq R\right) \\
& R_{2}=a R \\
& R_{3}=K(1-a) R \\
& R_{4}=\frac{K(1-a)}{K-1} R
\end{aligned}
$$

The total resistance for a specific gain $K$ is

$$
R_{t}=\left(1+a+\frac{K^{2}(1-a)}{K-1}\right) R
$$

From eqns. (8) and (11), we see that $R_{t} \leq R_{t 3}, R_{t 2}, R_{t 1}$ for all values of $a \leq 1$. 


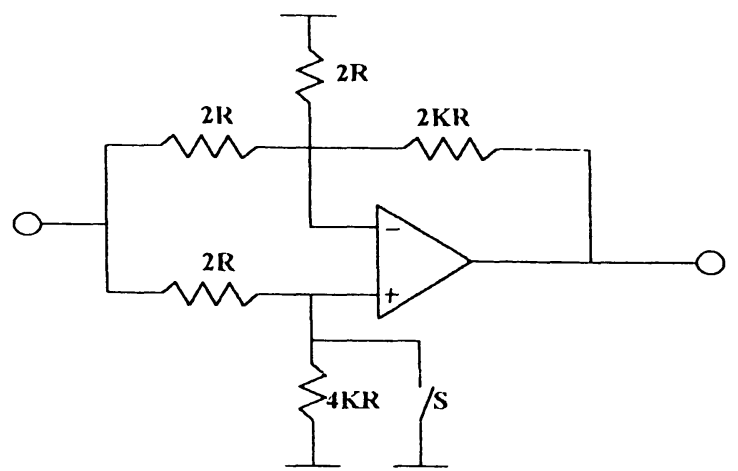

(a) Case (i)

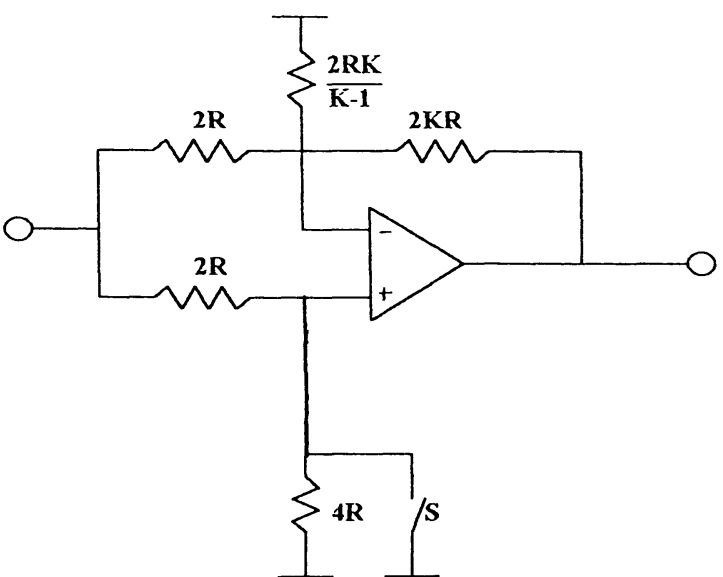

(b)

Case (ii)

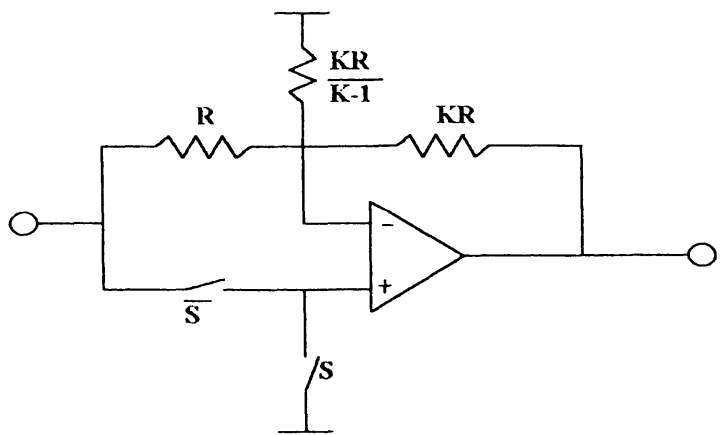

(c)

Case (iii)

FIGURE 2 Amplifier configurations reported in reference [1] 
Thus, the present circuit has the least value for $R_{t}$ for any specific gain $K$. However, $a$ cannot be 1 , because $a=1$ reduces $R_{3}=R_{4}=0$ and consequently, the output will be shorted. $R_{t}$ decreases with increase in $a$. Therefore, for minimum $R_{t}, a$ should be chosen as close as possible to unity. We choose $a=0.9$ to yield low $R_{t}$ and also reasonably small spread in resistance values given by eqn. (10).

The complete set of design relations are

$$
\begin{aligned}
& R_{1}=R, R_{2}=0.9 R, R_{3}=\frac{K R}{10}, R_{4}=\frac{K}{10(K-1)} R, R_{6}=0 \\
& R_{t}=\left(1.9+\frac{K^{2}}{10(K-1)}\right) R
\end{aligned}
$$

\section{IMPLEMENTATION}

Fig. 3 gives the amplifier with $N$ gain values programmed. It uses $(N+2)$ switches, one more than the minimum value. However, the on-resistances $R_{s}$ of the switches affect the gain accuracy. The effect can be made negligible by

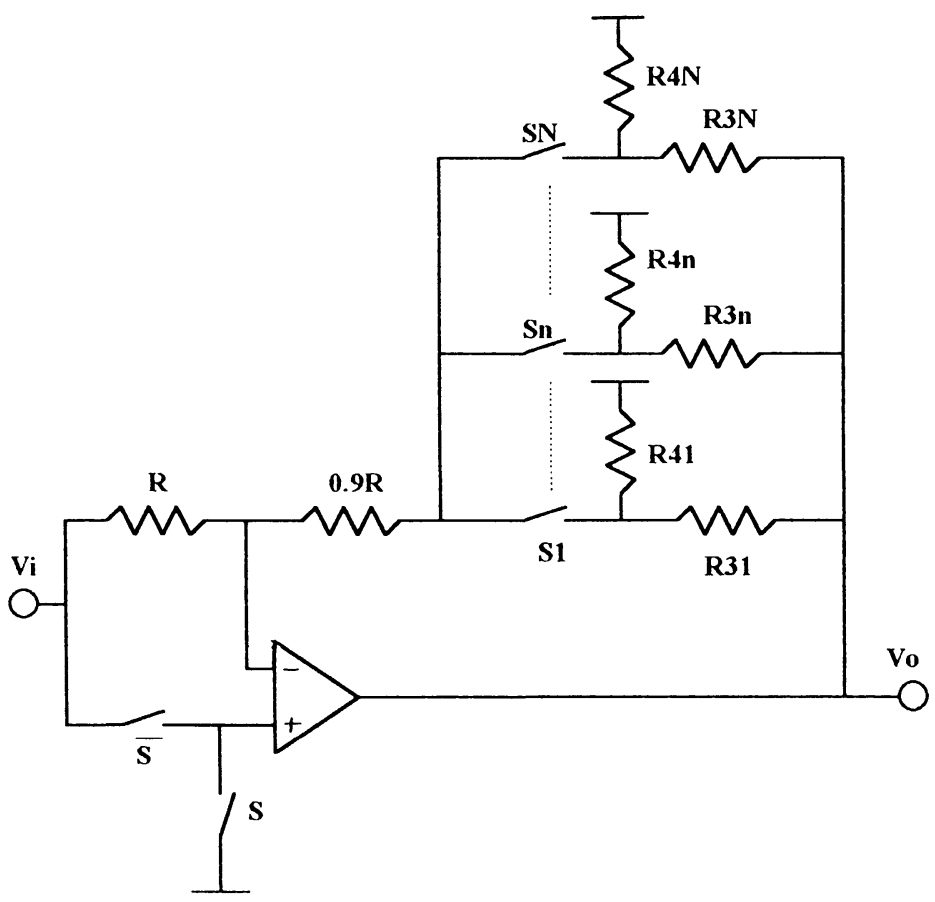

FIGURE 3 Amplifier implementation with less number of switches 
choosing the lowest resistance much higher than maximum $R_{s}$, but this means using high-valued resistances, increasing the total resistance. To fully compensate for the effect of $R_{s}$, an alternative implementation is given in Fig. 4. Now, each resistance has a switch in series. Thus, the effect of on-resistance of each switch can be compensated for by reducing the resistance value by the on-resistance of the switch in series with it.

Assuming that all the switches have equal drift-free on-resistance $R_{s}$, another circuit implementation is shown in Fig. 5 where

$r_{3 n}= \begin{cases}R_{3 n}-R_{3 n-1}, & 2 \leq n \leq N \\ R_{31}, & n=1\end{cases}$

$r_{4 n}=\left\{\begin{array}{lll}R_{4 n}-R_{4 n+1}, & 1 \leq n \leq N-1, & K_{1} \neq 1 \\ R_{4 n}-R_{4 n+1}, & 2 \leq n \leq N-1, & K_{1}=1 \\ \infty, & n=1, & K_{1}=1\end{array}\right.$

The total resistance of the circuit is now given by

$R_{t}=\left(1.9+\frac{K_{N}}{10}+\frac{K_{0}}{10\left(K_{0}-1\right)}\right) R$

where $K_{0}=K_{\min }>1, K_{n}>K_{n-1}(n=1,2, \cdots, N)$. To have a quantitative feel,

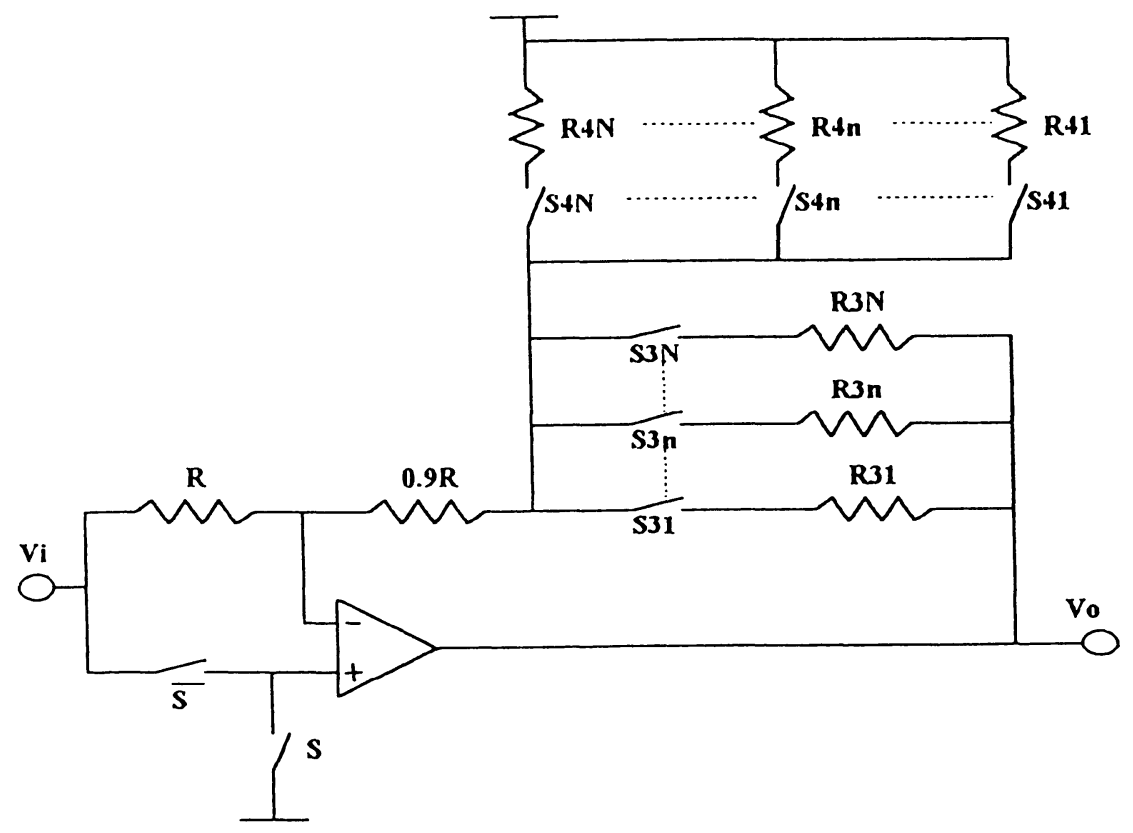

FIGURE 4 Fully compensated implementation of programmable amplifier 


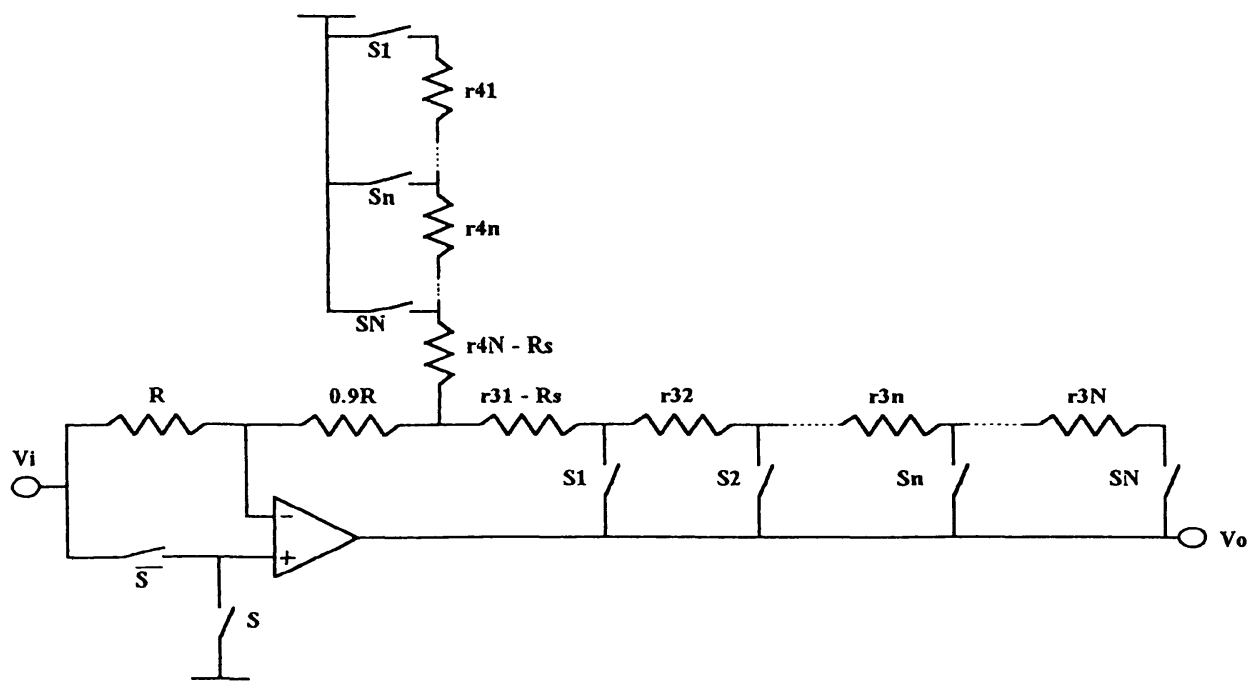

FIGURE 5 Compensated amplifier with reduced total resistance

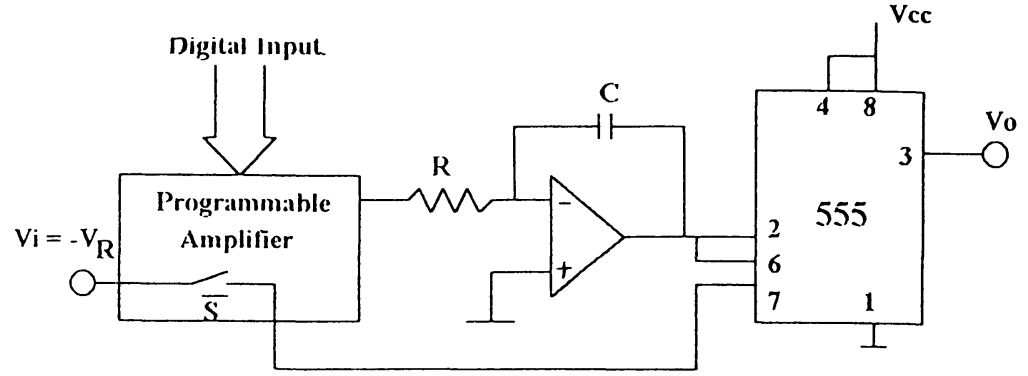

Switch $S$ is replaced by the internal switch of 555 .

(a)

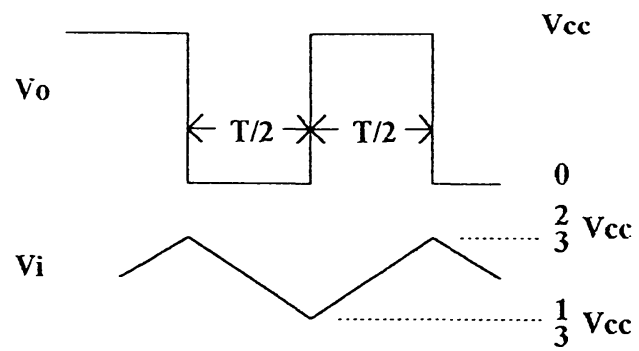

(i)

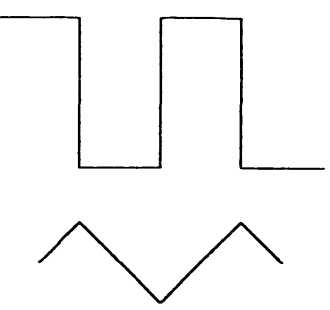

(ii)

(b)

FIGURE 6 (a) Digitally programmable function generator (b) Waveforms for (i) Digital input $D_{1}$ (ii) Digital input $D_{2}, D_{2}>D_{1}$. 
the $R_{t}$ for implementing 10 gain values (gain 1,2,10 and the remaining seven of arbitrary values lying between 2 and 10 ) and choosing $R=10 K$, eqn. (15) gives $R_{t}=31 K \Omega$.

\section{APPLICATION}

A typical application of a dual-polarity controlled amplifier is shown in Fig. 6 in realizing a function generator whose frequency can be digitally programmed.

\section{CONCLUSION}

A digitally programmable, dual-polarity gain amplifier has been presented. Though it uses more resistors, the total resistance is less than those in Ref. [1]. Thus, the former amplifier is superior to the latter ones for integration. The amplifier can be fully compensated for individual on-resistances of the switches (as shown in Fig. 4). If the on-resistance is assumed to be the same for all the switches, the total resistance can be further reduced (as shown in Fig. 5). An application of a dual-polarity programmable amplifier in realizing a programmable function generator has been given.

\section{ACKNOWLEDGMENTS}

The pre-competitive 1993 research grant and the research grand under Visiting Researcher 1993 Scheme by the University of South Australia are acknowledged.

\section{REFERENCE}

1. Rathore, T.S.: “Digitally controlled amplifiers with fewer resistors and sensitivities", Electron. Lett., 1983,19 , pp. 646-647. 

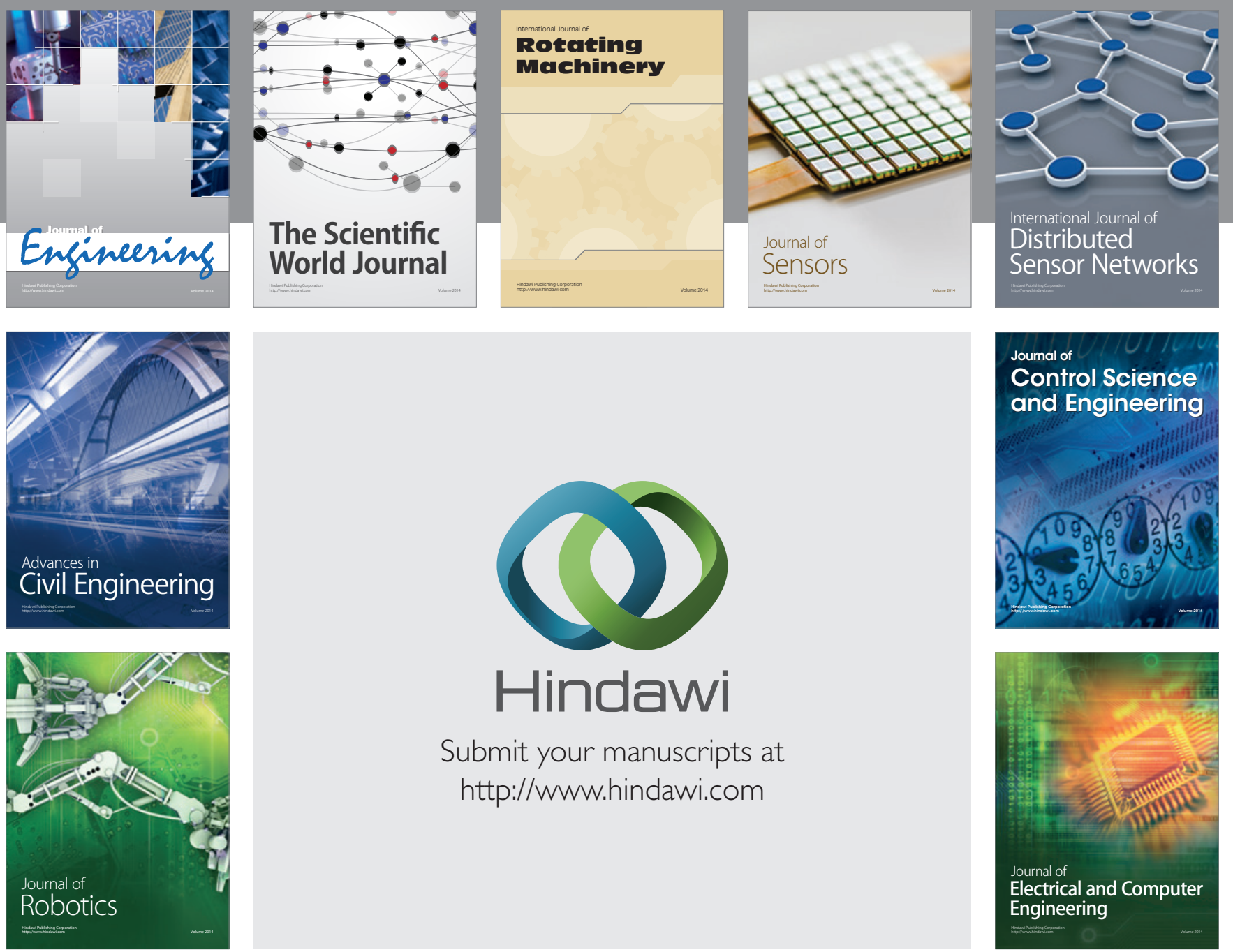

Submit your manuscripts at

http://www.hindawi.com
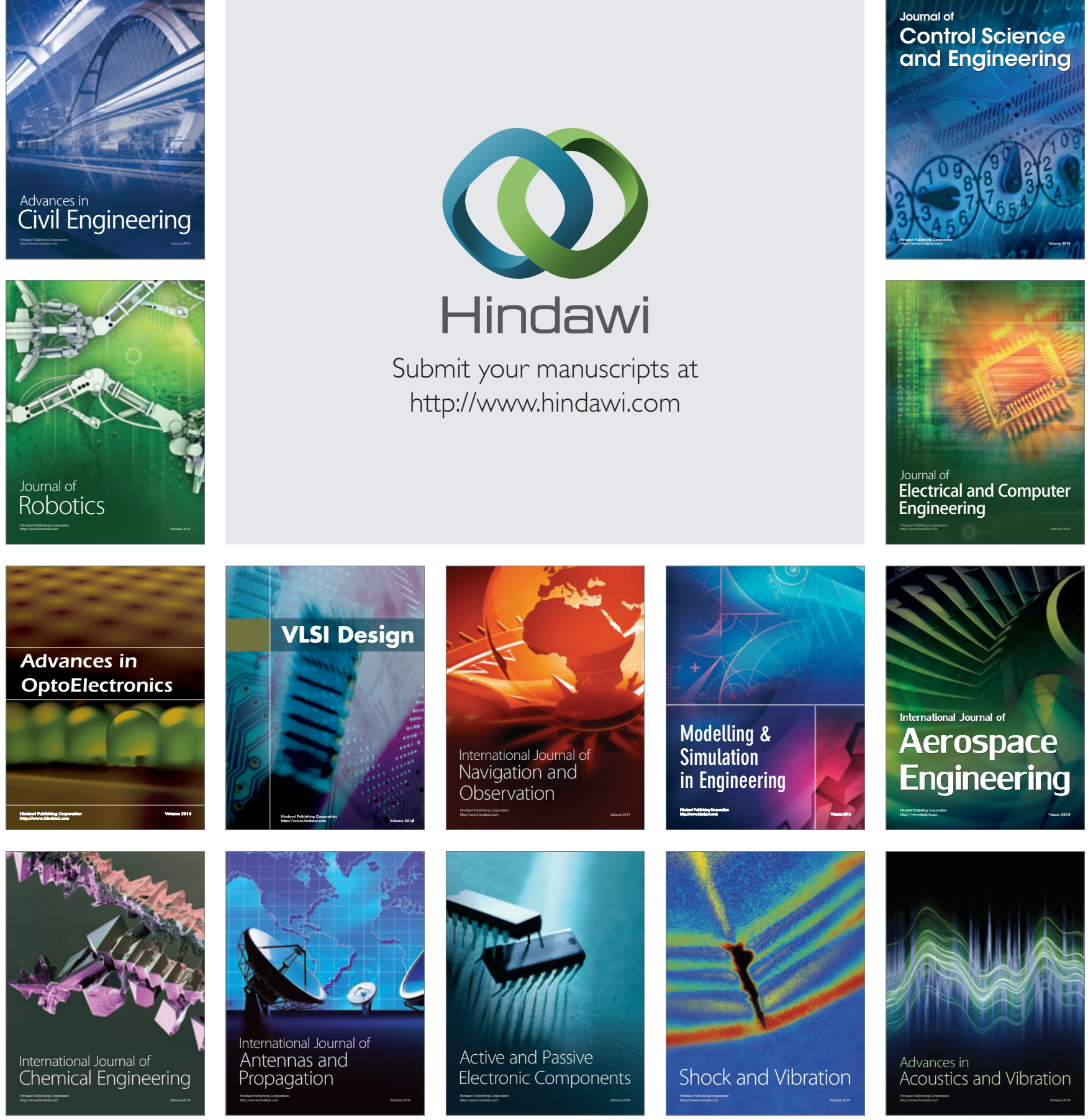